\title{
Arterial Dissection in Antiphospholipid Syndrome Patients: Two Case Reports and a Literature Review
}

\author{
Kadhim Al-banaa ${ }^{1}$, Alfarooq Alshaikhli ${ }^{2}$, Asal AL-Hareeri ${ }^{3}$, Mohammed Abdelhalim ${ }^{4}$, Alsadiq Al-Hillan ${ }^{5}$, Tejas Joshi ${ }^{6}$ \\ ${ }^{1}$ Department of Haemostasis and Thrombosis, University of California San Diego Health, San Diego, CA, USA \\ ${ }^{2}$ Department of Medicine, University of Texas/Rio Grande Valley, Edinburg, TX, USA \\ ${ }^{3}$ Department of Surgery, Harborview Medical Center, Seattle, WA, USA \\ ${ }^{4}$ Department of Medicine, Beaumont Hospital, Dearborn, MI, USA \\ ${ }^{5}$ Department of Gastroenterology, Beaumont Hospital, Royal Oak, MI, USA \\ ${ }^{6}$ Department Gastroenterology and Hepatology, Baylor College of Medicine, Houston, Texas, USA
}

\section{Doi: 10.12890/2021_002610- European Journal of Case Reports in Internal Medicine - ๑ EFIM 2021}

Received: 03/05/2021

Accepted: 07/05/2021

Published: $24 / 05 / 2021$

How to cite this article: Al-banaa K, Alshaikhli A, AL-Harreri A, Abdelhalim M, Al-Hillan A, Joshi T. Arterial dissection in antiphospholipid syndrome patients: two case reports and a literature review. EJCRIM 2021;8: doi:10.12890/2021_002610

Conflicts of Interests: The Authors declare that there are no competing interests.

This article is licensed under a Commons Attribution Non-Commercial 4.0 License

\section{ABSTRACT}

Antiphospholipid syndrome (APS) is a multisystemic autoimmune disease which presents with thromboembolic disease, pregnancy complications and the presence of antiphospholipid antibodies. There are some reports of arterial dissections in different sites of the body associated with APS. We describe two patients with APS who developed ischaemic stroke as a result of vertebral artery dissection in the absence of acquired and genetic risk factors for arterial dissection. We also conducted a systematic review of the literature for cases of arterial dissection associated with APS. We suspect that APS may be a potential cause of vasculopathy and arterial dissection. Further research is needed to explore this possible association.

\section{LEARNING POINTS}

- Antiphospholipid syndrome (APS) is a major risk factor for stroke in young patients.

- APS may be associated with vasculopathy and arterial dissection.

- Patients should meet both clinical and laboratory criteria for a diagnosis of APS.

\section{KEYWORDS}

Antiphospholipid antibody syndrome, APS, arterial dissection, vasculopathy, stroke

\section{INTRODUCTION}

Antiphospholipid syndrome (APS) is a multisystemic autoimmune disease with a high predilection for females. APS can be primary or secondary to other autoimmune disorders such as systemic lupus erythematosus (SLE). The prevalence of the disease is 1-5\% in the general population, but only a small proportion develop symptoms. The clinical manifestations of the disease differ among patients depending on the location of the vessel and the organ involved. The major presentations of APS include pregnancy-related comorbidities such as recurrent miscarriage, fetal death, preeclampsia, placental insufficiency and fetal growth restriction, in addition to multiple episodes of arterial, venous or small vessel thrombosis in different organs or tissues ${ }^{[1,2]}$.

Antiphospholipid antibodies are circulating immunoglobulins associated with high vascular risk for cardiovascular events that can affect both arteries and veins. Ischaemic stroke and transient ischaemic attacks are the most commonly described arterial thrombotic events. 
APS is characterized by the presence of circulation antibodies such as lupus anticoagulant (LAC), anti-cardiolipin (aCL), anti-B2 glycoprotein (A-B2GLP) and anti-prothrombin ${ }^{[3,4]}$.

A few case reports demonstrate a possible relationship between arterial dissection and APS. In this report, we present two patients with a previously established diagnosis of APS who presented later with a cerebrovascular accident (CVA) with concurrent vertebral artery dissection with abrupt onset of neurological sequelae. We also conducted a systematic review of the literature to explore the association between APS and arterial dissection.

\section{METHODS}

A systematic review of the literature was performed to identify cases of arterial dissection of any type and location in patients with APS. We searched PubMed and Google Scholar using the search terms 'antiphospholipid syndrome', 'aPL', 'arterial dissection', 'peripheral artery dissection', 'coronary artery dissection' and 'dissection'. Studies were included if they were published as original articles, case reports, letters or meeting abstracts. Review articles, meta-analyses, editorial comments and studies not fulfilling the inclusion criteria were excluded. In the selected studies, we also noted any relevant comorbid conditions and autoimmune disorders, therapy used and course of illness. We obtained written, informed consent from both patients for the publication of the details of their cases.

\section{Case 1}

The first patient was a 39-year-old woman who had received a diagnosis of triple-positive APS after a transient ischaemic attack when she was 26 years old. She presented to hospital with non-resolving occipital headache and right-sided paraesthesia that had started spontaneously with no preceding trauma or injury. The patient was not on anticoagulation or antiplatelet therapy. She did not have risk factors for atherosclerosis, was not a smoker or illegal drug user, and had no family history of stroke. On initial evaluation, the National Institutes of Health Stroke Scale (NIHSS) score was 1, and blood pressure and fundoscopic examination were normal. The chest radiograph, electrocardiogram, carotid duplex and all blood tests were normal. Brain magnetic resonance imaging (MRI) on admission did not demonstrate acute infarct or intracranial haemorrhage. Subsequent brain and neck magnetic resonance angiography (MRA) demonstrated right vertebral artery dissection, with absent contrast flow from the distal V2 segment through the basilar tip (Fig. 1). Warfarin was started following an initial period of heparin therapy. The patient's symptoms fully resolved within 48 hours.

Due to the lack of risk factors for arterial dissection, the patient underwent genetic testing for connective tissue disease including Marfan syndrome and all subtypes of Ehlers-Danlos syndrome. Tests were negative for any genetic mutation associated with connective tissue disease. Her previous work-up for APS revealed abnormal coagulation studies including a prolonged activated partial thromboplastin time of 40.2 $\mathrm{s}$ (normal range 25.1-37.6 s), which did not correct with 1:1 mixing with normal plasma, a thromboplastin inhibition test that was strongly positive for lupus anticoagulant, and a dilute Russell's viper venom test that was prolonged at $52.3 \mathrm{~s}$ (normal 29-46 s), which corrected with the addition of phospholipids. Her APS was characterized by substantial levels of immunoglobulin (Ig) G anticardiolipin antibodies (160 IgG antiphospholipid units (GPLU), reference <20) and IgM anticardiolipin antibodies (23 IgM antiphospholipid units (MPLU), reference <15), anti- $\beta 2$ glycoprotein 1 antibodies IgG (150 IgG units (SGU), reference <20) and anti- $\beta 2$ glycoprotein 1 antibodies IgM (22 IgM units (SGU), reference $<20)$. The abnormal laboratory findings were confirmed after they were repeated more than 12 weeks apart.

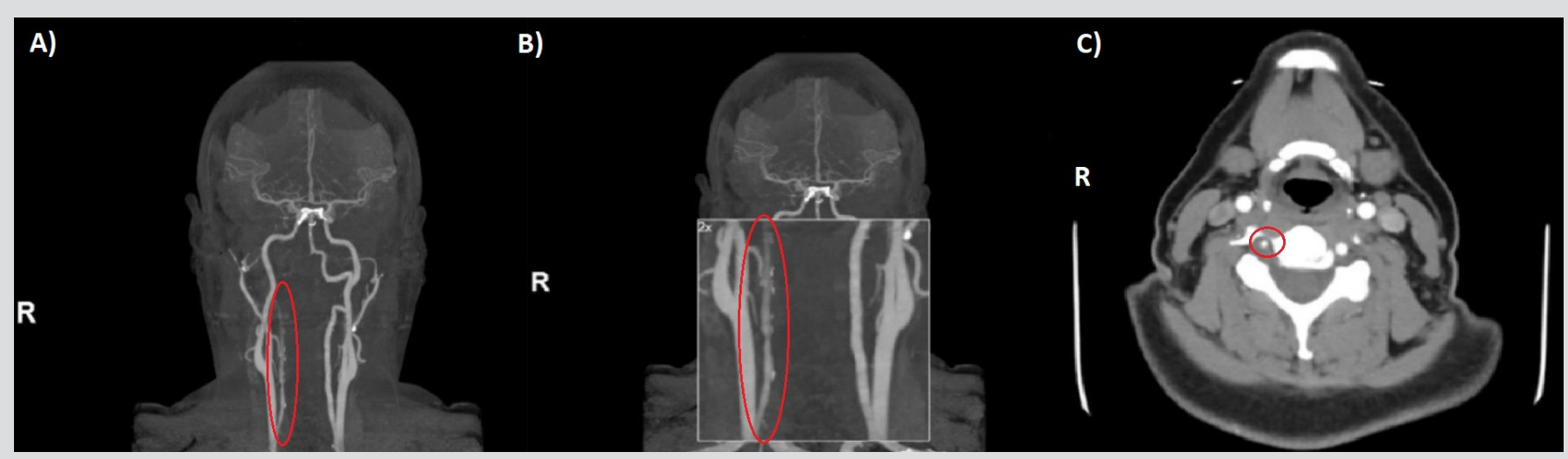

Figure 1. (A, B) Magnetic resonance angiography (MRA) coronal view, and (C) MRA cross-section at the level of C5 showing near occlusion/occlusion of the right vertebral artery with areas of intraluminal T1 hyperintensity, including an eccentric, circumferential mural focus reflecting intramural haematoma secondary to dissection 
Case 2

The second patient was a 39-year-old woman who had a history of APS that was diagnosed after an episode of unprovoked pulmonary embolus and recurrent miscarriages. The patient was on anticoagulation treatment with warfarin. She was referred to our centre after experiencing recurrent spontaneous vertebral artery dissections. She did not have risk factors for atherosclerosis, was not a smoker or illegal drug user, and did not have a family history of stroke. The first episode was diagnosed after multiple episodes of syncope, paraesthesia and occipital headache as brain magnetic resonance imaging (MRI) and computed tomography (CT) angiography suggested left vertebral artery dissection with cerebellar and right posterior cerebral artery (PCA) territory infarcts. These findings were confirmed later with an angiogram, which demonstrated occlusive dissection of the left vertebral artery at the level of C2. The second event was diagnosed when the patient was admitted with headache, neck stiffness, nausea, dizziness, blurry vision and aphasia. Brain and neck MRA demonstrated bilateral vertebral artery dissections. Blood pressure was normal on both admissions.

Testing for rheumatic disease with antinuclear antibody assay and anti-double stranded DNA (anti-dsDNA) was negative, and genetic testing for connective tissue disorders including Marfan syndrome and all subtypes of Ehlers-Danlos syndrome was also negative.

Previous work-up for APS revealed abnormally high levels of immunoglobulin (Ig) G anticardiolipin antibodies (25, 28 and 23 IgG antiphospholipid units (GPLU), reference <20) and IgM anticardiolipin antibodies (21, 22 and 19 IgM antiphospholipid units (MPLU), reference $<15$ ) on multiple occasions more than 12 weeks apart. Lupus anticoagulant and anti- $\beta 2$ glycoprotein 1 antibodies were negative.

\section{DISCUSSION}

Arterial dissection occurs when a tear develops in the intimal layer of an artery. Although uncommon, it is a major cause of stroke in young patients, particularly in the 35-45-year-old age group, and has a predilection for females. It is believed to account for $2.5 \%$ of cases of CVA in the general population and for 5-20\% in individuals younger than 45 years of age ${ }^{[5]}$. Andreoli et al. analysed 120 full-text papers published in English between the years 1984 and 2011, which showed that approximately 6\% of APS patients may develop pulmonary embolism, $13.5 \%$ stroke, $11 \%$ myocardial infarction, and $9.5 \%$ deep venous thrombosis. Many comorbid conditions can precipitate arterial dissection, such as hypertension, connective-tissue disorders, type IV Ehlers-Danlos syndrome, fibromuscular dysplasia, Marfan syndrome, autosomal dominant polycystic kidney disease, osteogenesis imperfecta, and the use of oral contraceptives ${ }^{[6,7]}$.

Many reported cases have suggested a correlation between APS and arterial dissection. Numerous pathophysiological effects of APS may precipitate dissection development. APS is characterized by a heterogeneous group of antibodies that exert major effects through binding to the phospholipid membrane of platelets leading to their activation. Moreover, antibodies attach to endothelium, monocytes and neutrophils, resulting in a hypercoagulability effect. In addition, APS potentiates complement system activation and causes type III hypersensitivity. Furthermore, in vivo and in vitro studies show that anticardiolipin antibodies have an atherogenic role prompting thickening of the carotid intima and possibly interfering with wall remodelling in patients with APS. Collectively, these factors increase the risk of coagulation and vascular alteration ${ }^{[8,9]}$.

In Table 1, we list cases of dissection in patients diagnosed with APL. The clinical characteristics of the 19 patients identified through the literature review included young age at dissection detection (average age of 36.68 , youngest: 23 , oldest: 61 years of age at presentation) and female preponderance (13 cases, 68.42\%). The atherosclerotic vascular risk factors of hypertension, smoking and peripheral artery disease were seen in three patients ( $n=3$ for each, 15.7\%), but other vascular risk factors including diabetes mellitus, dyslipidaemia and illicit drug abuse, were not found.

Co-existing autoimmune diseases included SLE in five patients (26.3\%), Takayasu arteritis in two patients (10.5\%), and rheumatoid arthritis, autoimmune hepatitis and autoimmune hypothyroidism in one patient each (5.2\%). The following positive antiphospholipid antibodies were detected: aCL in 14 patients (73.68\%), LAC in 9 patients (47.3\%), A-B2GLP in 6 patients (31.5\%) and aPE in 1 patient (5.2\%). The different vessels involved included the internal carotid artery, aorta and coronary arteries in four patients each (21.05\%), the vertebral artery and cerebral arteries in three patients (15.7\%), and the superficial femoral artery in one patient (5.2\%) ${ }^{[10-25]}$.

In this review, we describe two cases of CVA resulting from dissection of the posterior cerebral artery. Both patients met the clinical and laboratory criteria for APS (Table 2). The first patient was diagnosed with APS after a TIA and confirmed laboratory tests showing triple positive APS antibodies IgG anticardiolipin antibodies, IgM anticardiolipin antibodies and anti- $\beta 2$ glycoprotein antibodies IgG confirmed on multiple occasions 12 weeks apart. The second patient had recurrent miscarriages and positive immunoglobulin IgG anticardiolipin antibodies and IgM anticardiolipin antibodies on multiple occasions more than 12 weeks apart. Both were diagnosed after other potential differential diagnoses had been eliminated and potential risk factors excluded ${ }^{[26]}$.

Having obtained detailed histories and conducted physical examinations concurrently with genetic studies and autoimmune disease workup, we theorize that APS might be the cause of the vertebral artery dissections, supported by the absence of atherosclerotic risk factors in both patients, who were also negative for SLE. 


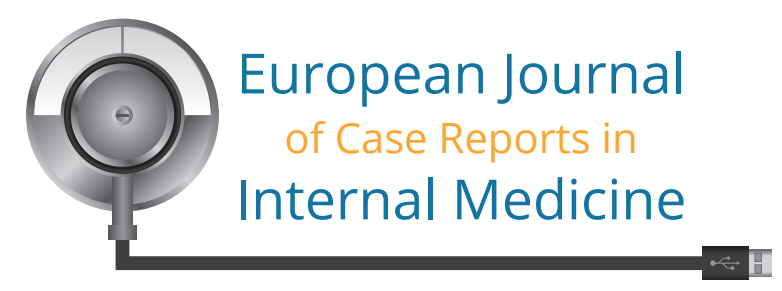

\begin{tabular}{|c|c|c|c|c|c|c|c|c|c|c|c|c|}
\hline 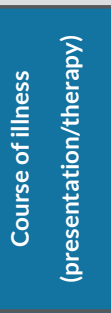 & 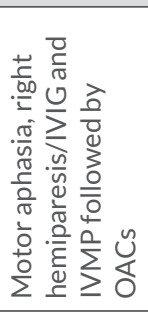 & $\underset{z}{\longleftarrow}$ & $\underset{z}{\nwarrow}$ & 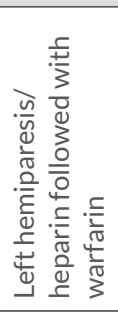 & 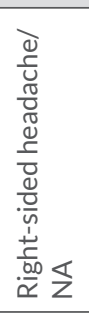 & 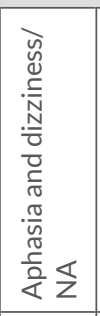 & 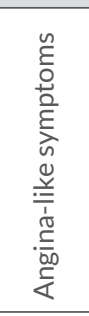 & 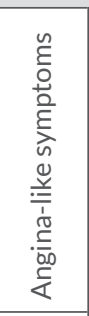 & 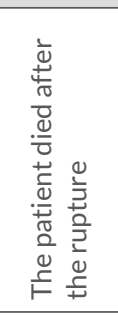 & 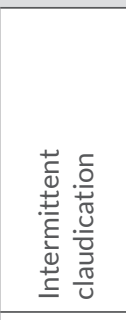 & 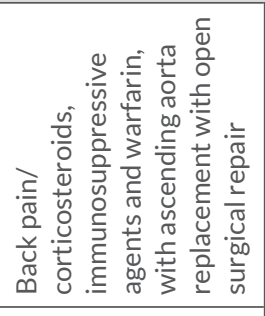 & 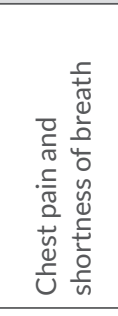 \\
\hline 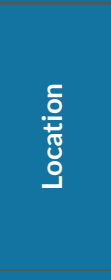 & 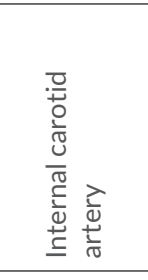 & 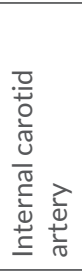 & 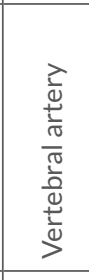 & 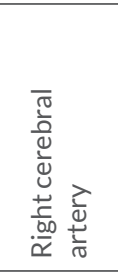 & 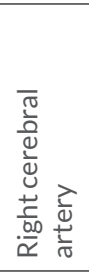 & 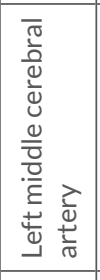 & 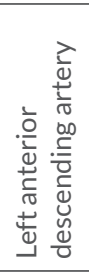 & 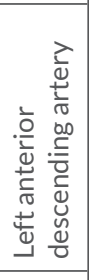 & 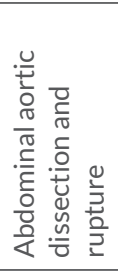 & 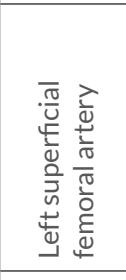 & 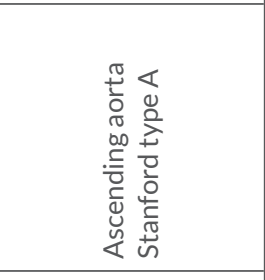 & 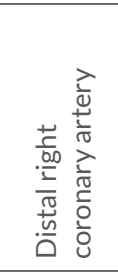 \\
\hline 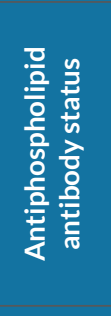 & 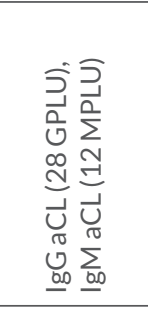 & 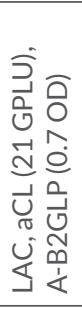 & 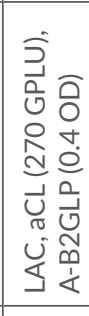 & 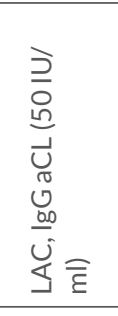 & 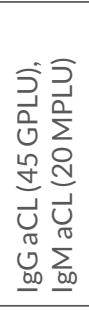 & 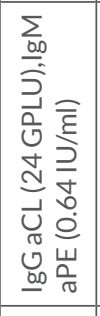 & $\underset{\widetilde{\sigma}}{\vec{U}}$ & $\underset{\sigma}{\vec{J}}$ & 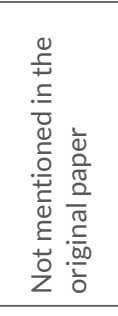 & 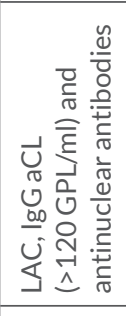 & 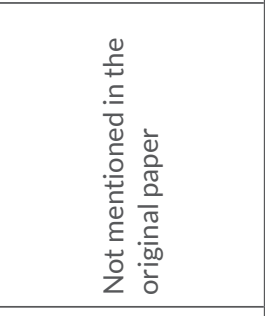 & 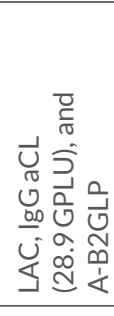 \\
\hline 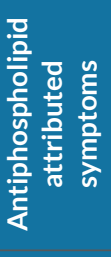 & 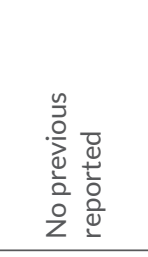 & 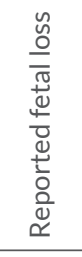 & 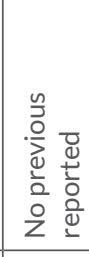 & 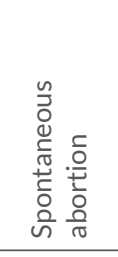 & 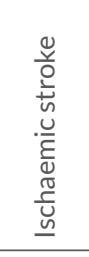 & 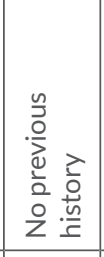 & 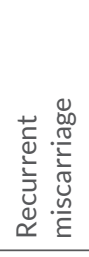 & 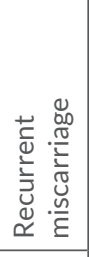 & 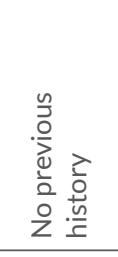 & 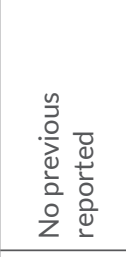 & 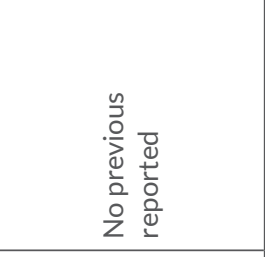 & 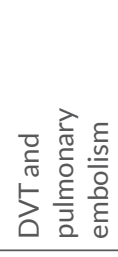 \\
\hline 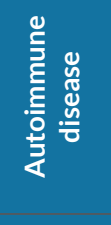 & $\begin{array}{l}0 \\
\text { Oे }\end{array}$ & $\begin{array}{l}\text { ¿̃ } \\
\text { ż }\end{array}$ & $\begin{array}{l}0 \\
\text { ò } \\
\text { z }\end{array}$ & 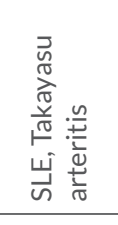 & 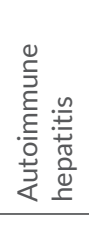 & $\begin{array}{l}0 \\
\text { ò }\end{array}$ & $\begin{array}{l}0 \stackrel{0}{0} \\
\text { Zे }\end{array}$ & 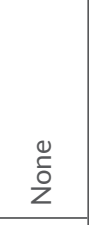 & 山্ & 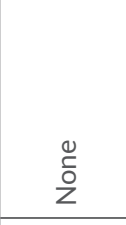 & $\underset{\sim}{\omega}$ & 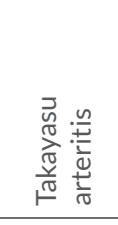 \\
\hline 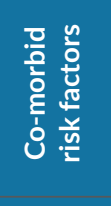 & $\begin{array}{l}0 \\
\stackrel{0}{0} \\
z\end{array}$ & $\begin{array}{l}\frac{\tilde{\sigma}}{0} \\
\text { ż }\end{array}$ & $\begin{array}{l}0 \\
\text { ¿े } \\
\text { z }\end{array}$ & 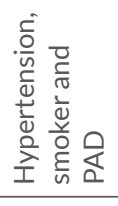 & $\begin{array}{l}0 \\
\text { ô } \\
\text { z }\end{array}$ & $\begin{array}{l}0 \\
\text { ô } \\
\text { z }\end{array}$ & $\begin{array}{l}\text { Ĩ } \\
\text { ż }\end{array}$ & $\begin{array}{l}\mathscr{0} \\
\text { ż }\end{array}$ & $\begin{array}{l}0 \\
\text { ¿े } \\
\text { z }\end{array}$ & 足 & $\begin{array}{l}\tilde{\Sigma} \\
\text { Zे }\end{array}$ & $\begin{array}{l}\tilde{0} \\
\text { o }\end{array}$ \\
\hline 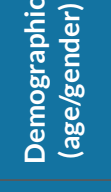 & 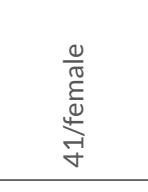 & 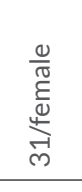 & 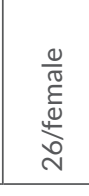 & 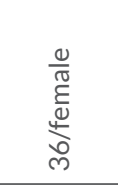 & 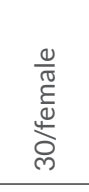 & 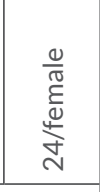 & 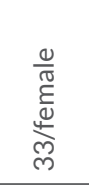 & 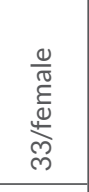 & 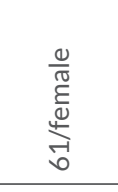 & 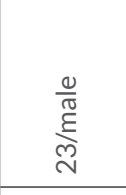 & 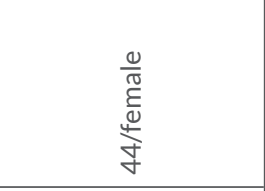 & 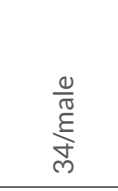 \\
\hline 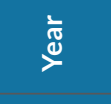 & $\stackrel{g}{g}$ & $\xi$ & 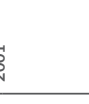 & ఫ్ & ఏ్ & 苦 & ఫ్ & : & 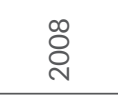 & ठे & $\begin{array}{l}\text { ㅇ. } \\
\text { مे }\end{array}$ & $\stackrel{\infty}{\stackrel{N}{N}}$ \\
\hline 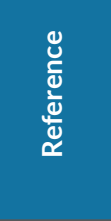 & 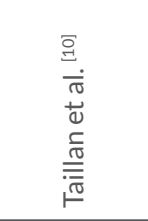 & & 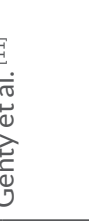 & 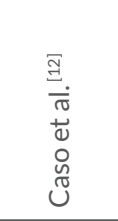 & 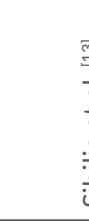 & 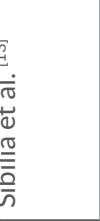 & 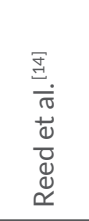 & 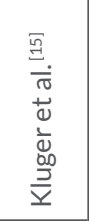 & 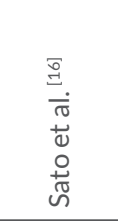 & 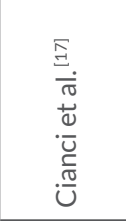 & 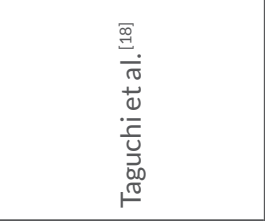 & 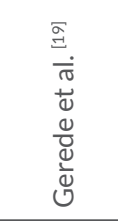 \\
\hline
\end{tabular}

Table 1 Previously reported cases of arterial dissection in patients diagnosed with antiphospholipid syndrome 


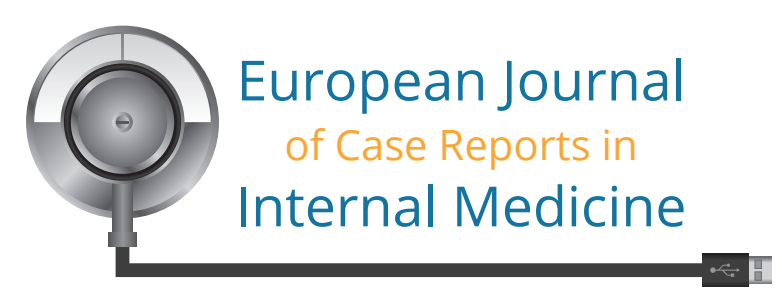

\begin{tabular}{|c|c|c|c|c|c|c|c|c|c|c|}
\hline 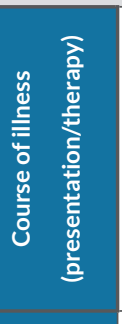 & 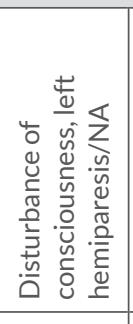 & 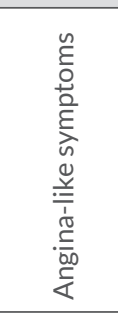 & 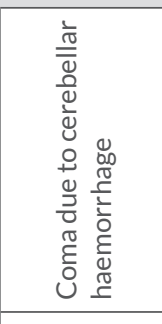 & 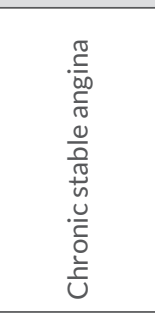 & 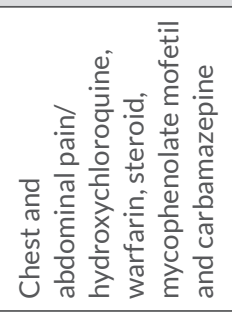 & 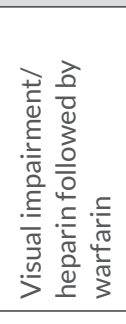 & 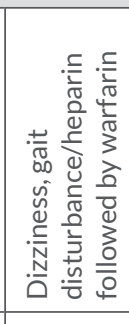 & 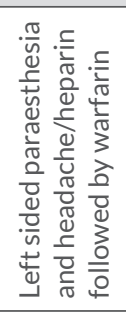 & 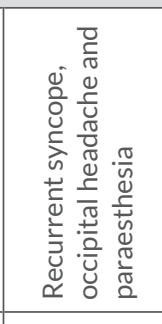 & 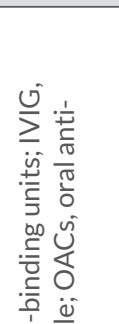 \\
\hline 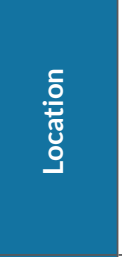 & 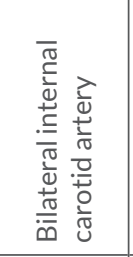 & 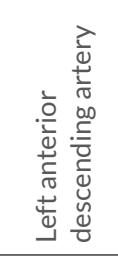 & 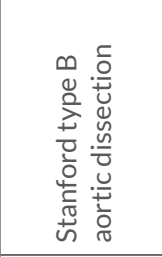 & 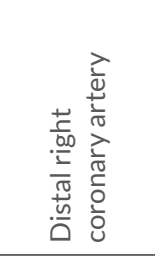 & 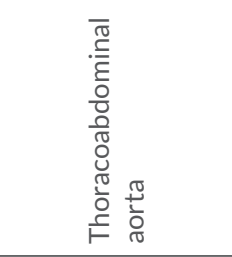 & 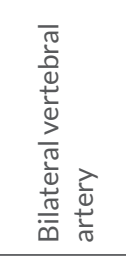 & 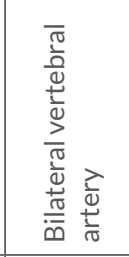 & 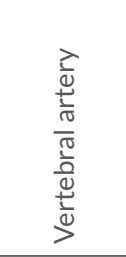 & 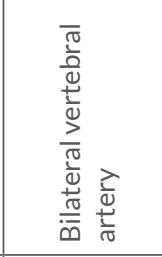 & 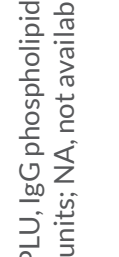 \\
\hline 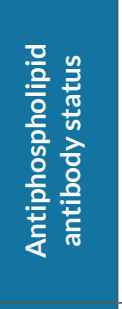 & U & 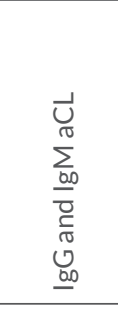 & 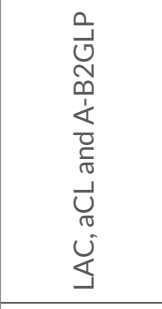 & 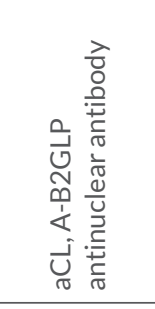 & $\begin{array}{l}\vec{U} \\
\tilde{T} \\
0 \\
\overline{0} \\
\bar{J}\end{array}$ & 岁 & 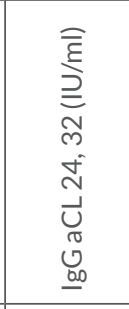 & 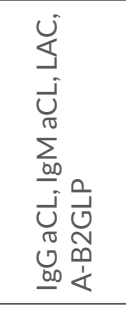 & 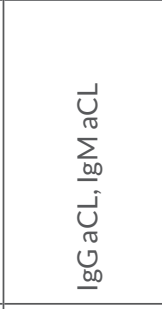 & 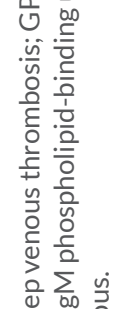 \\
\hline 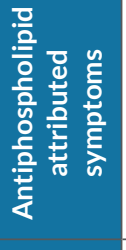 & 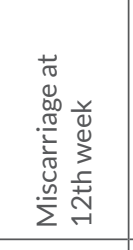 & 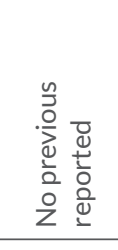 & 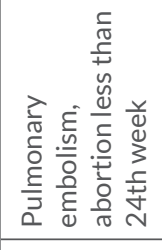 & 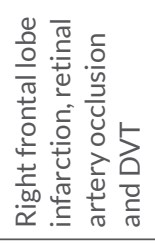 & 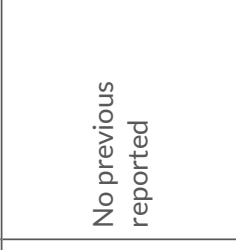 & 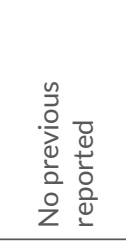 & 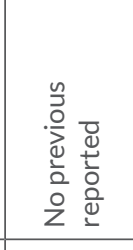 & 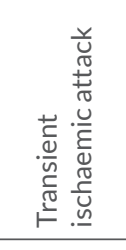 & 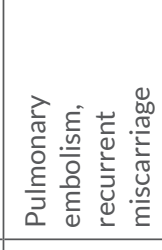 & 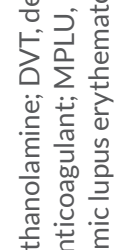 \\
\hline 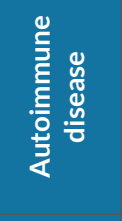 & $\begin{array}{l}0 \\
\text { ¿े }\end{array}$ & $\begin{array}{l}0 \\
\text { ò }\end{array}$ & $\begin{array}{l}0 \\
\text { ò } \\
z\end{array}$ & 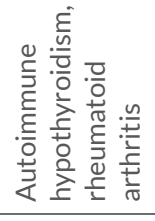 & 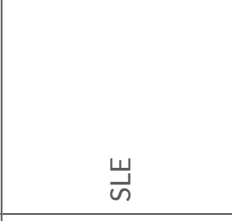 & $\underset{\omega}{\omega}$ & $\begin{array}{l}\text { ¿̊ } \\
\text { z }\end{array}$ & $\begin{array}{l}0 \\
\text { ò }\end{array}$ & $\begin{array}{l}0 \\
\text { ò }\end{array}$ & 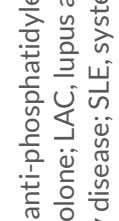 \\
\hline 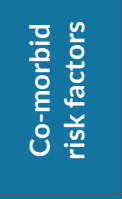 & $\begin{array}{l}0 \\
\text { I0 } \\
\text { z }\end{array}$ & 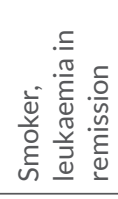 & 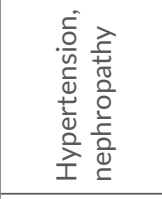 & 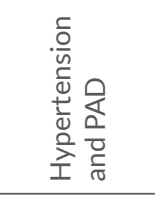 & 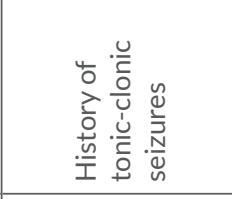 & $\begin{array}{l}0 \\
\text { ¿ } \\
z\end{array}$ & $\begin{array}{l}\tilde{U} \\
\text { Zे }\end{array}$ & $\begin{array}{l}\mathscr{0} \\
\stackrel{0}{z}\end{array}$ & $\begin{array}{l}0 \\
\text { ô } \\
\text { z }\end{array}$ & 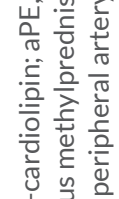 \\
\hline 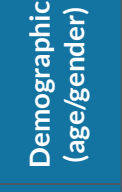 & 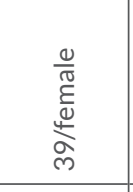 & $\begin{array}{l}\frac{0}{\tilde{\sigma}} \\
\frac{\hat{\Sigma}}{\mathrm{N}} \\
\text { }\end{array}$ & 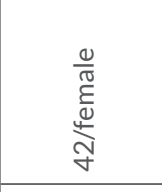 & $\begin{array}{l}\frac{0}{\tilde{\sigma}} \\
\underset{\text { N }}{\mathrm{N}}\end{array}$ & $\begin{array}{l}\frac{0}{\tilde{\sigma}} \\
\frac{\xi}{\sigma} \\
\text { ले }\end{array}$ & 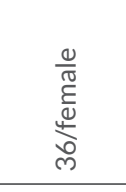 & 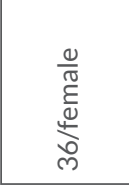 & 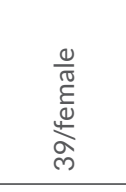 & 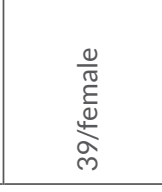 & 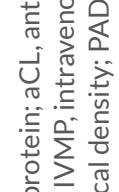 \\
\hline 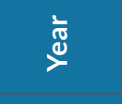 & $\stackrel{+}{\vec{N}}$ & $\stackrel{\infty}{\stackrel{\sim}{N}}$ & مे & مे & 尺ัญ & $\xi$ & Q & & ્ָ & $\frac{\mathrm{x}}{\mathrm{b}}$ \\
\hline 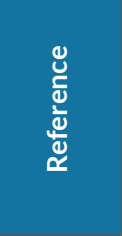 & 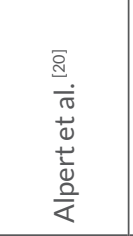 & 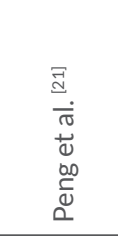 & 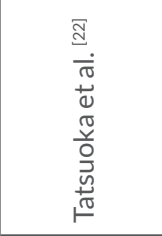 & 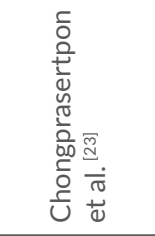 & 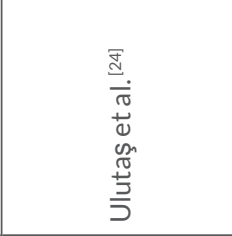 & & $\frac{\pi}{\bar{\sigma}}$ & 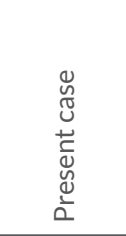 & 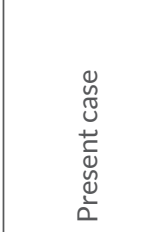 & 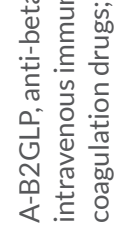 \\
\hline
\end{tabular}

Table 1 Previously reported cases of arterial dissection in patients diagnosed with antiphospholipid syndrome 
To the best of our knowledge, we believe these are the first two reported cases of APS patients presenting with PCA ischaemic stroke due to vertebral dissection who tested negative for SLE. Furthermore, this report adds to the existing literature on vasculopathy and identifies this area as a subject for further research.

\section{Clinical criteria (at least one of the following):}

a) Thrombosis: One or more episodes of arterial, venous or small vessel thrombosis, in any organ or tissue.

b) Pregnancy morbidity: One of the following:

1. One or more unexplained deaths of a morphologically normal fetus at or beyond the 10th week of gestation, with normal fetal morphology documented by ultrasound or by direct examination of the fetus.

2. One or more premature births of a morphologically normal neonate before the 34th week of gestation because of: eclampsia or severe preeclampsia defined according to standard definitions, or recognized features of placental insufficiency.

3. Three or more unexplained consecutive spontaneous abortions before the 10th week of gestation, with maternal anatomic or hormonal abnormalities and paternal and maternal chromosomal causes excluded.
Laboratory criteria (at least one of the following):

a) Positive lupus anticoagulant present in plasma, on two or more occasions at least 12 weeks apart.

b) IgG or IgM isotypes of anticardiolipin in serum or plasma, present in medium or high titre (i.e., > 40 lgG phospholipid units and/or IgM phospholipid units, or >the 99th percentile titre), present on two or more occasions, at least 12 weeks apart.

c) IgG or IgM isotypes of anti-beta-2 glycoprotein-I antibody in serum or plasma (in titre >the 99th percentile), present on two or more occasions, at least 12 weeks apart.

Table 2. Previously reported cases of arterial dissection in patients diagnosed with antiphospholipid syndromecircumferential mural focus reflecting intramural haematoma secondary to dissection

\section{CONCLUSION}

The precise mechanism behind vasculopathy and its consequences in patients with APS remains to be clearly defined. Vasculopathy has manifold presentations such as thrombosis, embolus, cutaneous manifestations, and pregnancy-related morbidity. Moreover, we demonstrate a potential relationship between APS and arterial dissection. Comorbid risk factors and coexisting autoimmune disease were present in some cases, which requires further research and study. Finally, the striking presentation of arterial dissection should be kept in mind by clinicians in patients diagnosed with or suspected of having APS. 


\section{REFERENCES}

1. Ruiz-Irastorza G, Crowther M, Branch W, Khamashta MA. Antiphospholipid syndrome. Lancet 2010;376(9751):1498-1509.

2. Branch DW, Eller AG. Antiphospholipid syndrome and thrombosis. Clin Obstet Gynecol 2006;49(4):861-874.

3. Whitaker KL. Antiphospholipid antibody syndrome: the difficulties of diagnosis. JAAPA 2017;30(12):10-14.

4. Bradacova P, Slavik L, Ulehlova J, Skoumalova A, Ullrychova J, Prochazkova J, et al. Current promising biomarkers and methods in the diagnostics of antiphospholipid syndrome: a review. Biomedicines 2021;9(2):166.

5. Grond-Ginsbach C, Pjontek R, Aksay SS, Hyhlik-Dürr A, Böckler D, Gross-Weissmann ML. Spontaneous arterial dissection: phenotype and molecular pathogenesis. Cell Mol Life Sci 2010;67(11):1799-1815.

6. Fullerton HJ, Johnston SC, Smith WS. Arterial dissection and stroke in children. Neurology 2001;57(7):1155-1160.

7. Andreoli L, Chighizola CB, Banzato A, Pons-Estel GJ, de Jesus GR, Erkan D. Estimated frequency of antiphospholipid antibodies in patients with pregnancy morbidity, stroke, myocardial infarction, and deep vein thrombosis: a critical review of the literature. Arthritis Care Res (Hoboken) 2013;65(11):1869-1873.

8. Khoynezhad A, White RA. Pathogenesis and management of retrograde type A aortic dissection after thoracic endovascular aortic repair. Ann Vascular Surg 2013;27(8):12011206.

9. Lelong DC, Logak M. Pathogenesis of spontaneous cervico-cerebral artery dissection. A hypothesis and a review of the literature. Med Hypotheses 2004;62(3):453-457.

10. Taillan B, Garnier G, Dujardin P. Dissection of the internal carotid artery in antiphospholipid syndrome. Rev Med Interne 1999;20(11):1048.

11. Genty I, Piette JC, Vandamme X, Samson Y, Chapman J, Papo T. Antiphospholipid antibodies in cervical artery dissection. Arthritis Rheum 2001;44(9):S148.

12. Caso V, Paciaroni M, Parnetti L, Cardaioli G, Biscarini L, Acciarini AE, et al. Stroke related to carotid artery dissection in a young patient with Takayasu arteritis, systemic lupus erythematosus and antiphospholipid antibody syndrome. Cerebrovasc Dis 2002;13(1):67-69.

13. Sibilia J, Hercelin D, Gottenberg JE, Goetz J, Dietemann JL, Harlé JR, et al. Cervico-cranial artery dissection and antiphospholipid syndrome: is there a link? Am J Med 2004;116(2):138-139.

14. Reed RK, Malaiapan Y, Meredith IT. Spontaneous coronary artery dissection in a female with antiphospholipid syndrome. Heart Lung Circ $2007 ; 16(2): 120-122$.

15. Kluger BM, Hughes RL, Anderson CA, Hassell KL. Non-traumatic carotid dissection and stroke associated with anti-phospholipid antibody syndrome: report of a case and review of the literature. Neurol India 2008;56(1):77.

16. Sato J, Kawakami T, Nakabayashi K, Fukuoka K, Hirano K, Terado Y, et al. Multiple aortic aneurysms complicated by a rupture in the systemic lupus erythematosus: a case report. Pathol Res Pract 2008;204(11):845-850.

17. Cianci R, Gigante A, Barbano B, Zaccaria A, Polidori L, Borghesi F, et al. Multiple steno-obstructive vascular lesions and femoral superficial artery dissection in a young Caucasian male with antiphospholipid syndrome. BMJ Case Rep 2009;2009:bcr1220081342.

18. Taguchi T, Maeba S, Watanabe K. Ascending aortic replacement for acute type A aortic dissection in a patient with anti-phospholipid antibody syndrome. Japan J Cardiovasc Surg 2010;39(5):265-268.

19. Gerede DM, Yüksel B, Tutar E, Küçükşahin O, Uzun Ç, Atasoy KÇ, et al. Spontaneous coronary artery dissection in a male patient with Takayasu's arteritis and antiphospholipid antibody syndrome. Case Rep Rheumatol 2013;2013:272963.

20. Alpert JN, White M, Perusquia E. Lupus anticoagulant associated with extracranial internal carotid artery occlusion. Stroke 1990;21(12):1759-1760.

21. Peng X, Zhu Z, Tang J, Zhou S. Spontaneous coronary artery dissection accompanied with antiphospholipid syndrome and leukemia. Int Heart J 2018:59(4):891-894.

22. Tatsuoka Y, Mano Y, Ishikawa S, Shinozaki S. Primary antiphospholipid antibody syndrome complicated with cerebellar hemorrhage and aortic dissection: a case report. Am $J$ Case Rep 2019;20:1852.

23. Chongprasertpon N, Ibrahim A, Goggins M, Kiernan T. Chronic spontaneous coronary artery dissection in association with antiphospholipid syndrome presenting as stable angina. BMJ Case Rep 2019;12(3):e227674.

24. Ulutaş F, Çobankara V, Bozdemir A, Karasu U. A rare association of antiphospholipid antibody syndrome, systemic lupus erythematosus and aortic dissection: a striking presentation with multi-organ failure? Eur J Case Rep Intern Med 2020;7(11):001887.

25. Iseki T, Yamashita Y, Ueno Y, Hira K, Miyamoto N, Yamashiro K, et al. Cerebral artery dissection secondary to antiphospholipid syndrome: a report of two cases and a literature review. Lupus 2021;30(1):118-124.

26. Miyakis S, Lockshin MD, Atsumi T, Branch DW, Brey RL, Cervera RH, et al. International consensus statement on an update of the classification criteria for definite antiphospholipid syndrome (APS). J Thromb Haemost 2006;4(2):295-306. 